\title{
CircRNA DUSPI6 Knockdown Suppresses Colorectal Cancer Progression by Regulating the miR-432-5p/E2F6 Axis
}

\section{Guangyao Wang \\ Haojun Yang}

Department of Gastrointestinal Surgery, The Affiliated Changzhou No.2 People's Hospital of Nanjing Medical University, Changzhou, 213000, People's Republic of China
Correspondence: Haojun Yang The Affiliated Changzhou No.2 People's Hospital of Nanjing Medical University, No. 68, Gehu Road, Changzhou, 213000 , People's Republic of China Tel +865I98I087286

Email yaojunyang82@I26.com
Background: Circular RNAs (circRNAs) play critical roles in various types of cancer. The aim of the present study was to investigate the function and underlying mechanism of CircRNA DUSP16 (circDUSP16) in colorectal cancer (CRC) development.

Materials and Methods: The expression levels of circDUSP16, microRNA-432-5p (miR432-5p), and E2F transcription factor 6 (E2F6) were measured by RT-qPCR. Cell proliferation, migration, invasion, and apoptosis were investigated by CCK-8, Transwell, and flow cytometry assays. Western blot analysis was used to evaluate the levels of the pro-apoptotic protein (Bax and cleaved-caspase 3) and the anti-apoptotic protein (Bcl-2). Luciferase reporter assay and RIP assay were used to analyze the association between miR-432-5p and circDUSP16 or E2F6. A xenograft tumor model was employed to explore the effect of circDUSP16 on CRC tumor growth in vivo.

Results: CircDUSP16 expression was upregulated in CRC tissues and cell lines. High circDUSP16 expression was correlated with low survival rate. Furthermore, circDUSP16 knockdown repressed cell proliferation, migration, and invasion and induced apoptosis in CRC. CircDUSP16 caused a negative regulation in miR-432-5p expression. In addition, E2F6 expression was elevated in CRC tissues. Inhibition of miR-432-5p promoted the proliferative and metastatic activity of CRC cells and inhibited the induction of apoptosis. Inhibition of E2F6 expression partially abolished the effects caused by miR-432-5p depletion. Moreover, circDUSP16 upregulated E2F6 expression by reducing miR-432-5p expression. Furthermore, circDUSP16 silencing repressed CRC tumor growth in vivo.

Conclusion: The results supported the hypothesis that circDUSP16 knockdown suppressed CRC progression by regulating the miR-432-5p/E2F6 axis, suggesting that the circDUSP16/ miR-432-5p/E2F6 network may be a potential therapeutic target for CRC.

Keywords: circDUSP16, miR-432-5p, E2F6, colorectal cancer, progression

\section{Introduction}

Colorectal cancer (CRC) is one of the most common and fatal malignancies and is considered as a leading cause of cancer-associated mortality for both men and women worldwide. ${ }^{1}$ Although significant progress has been made in the development of early diagnostic and treatment methods for $\mathrm{CRC}$, the prognosis of CRC patients remains disappointing. ${ }^{2}$ Due to metastasis and postoperative recurrence, the overall survival of CRC patients remains unsatisfactory and the 5-year survival rate is $<70 \%{ }^{3,4} \mathrm{CRC}$ is characterized by a multi-stage development process, which involves a variety of oncogenes and tumor suppressor genes. ${ }^{5}$ Therefore, the 
molecular mechanisms involved in CRC progression and development require further investigation.

Circular RNAs (circRNAs) are defined as a type of stable and covalent closed-loop RNA transcripts. As revealed by previous studies, circRNAs are deeply involved in the progression of human diseases, especially cancers; moreover, due to their stability and abundance, circRNAs may also serve as biomarkers for cancers. ${ }^{6,7}$ Several lines of evidence have shown that circRNAs can participate in pathological and physiological processes of cancer development, including lung, gastric, pancreatic, and colorectal cancers. ${ }^{8-10}$ CircRNA DUSP16 (CircDUSP16), a newly discovered circRNA, has been shown to function as an oncogene in several cancer types. For example, circDUSP16 facilitates gastric cancer tumorigenesis and invasion as a sponge of miR-145-5p. ${ }^{11}$ Besides, CircDUSP16 exacerbates hypoxia-induced esophageal squamous cell carcinoma cell growth, invasion, and glycolysis by upregulating TKTL1 via miR-497-5p. ${ }^{12}$ It was also reported that circDUSP16 was upregulated in $\mathrm{CRC},{ }^{13}$ implying its oncogenic role in CRC. However, the specific functions of circDUSP16 in CRC progression remain to be determined.

miRNAs are a class of short non-coding RNAs (19-22 nts) that may cause cleavage or suppress the translation of mRNAs by targeting their 3 '-UTR. ${ }^{14}$ A number of studies have shown that various miRNAs participate in the progression of certain types of cancer, including CRC. For example, miR-539 suppressed the progression of human CRC by regulating RUNX2. ${ }^{15}$ miR-3150b-3p repressed CRC progression by downregulating GOLPH3 expression. ${ }^{16}$ miR-199a-5p was shown to inhibit CRC development by regulating ROCK $1 .{ }^{17}$ miR-432-5p has been reported to act as a tumor suppressor in various cancer, such as breast cancer, ${ }^{18}$ hepatocellular carcinoma, ${ }^{19}$ and pancreatic cancer. ${ }^{20}$ Recently, Luo et al revealed that miR-432-5p inhibited the cell migration and invasion of CRC. ${ }^{21}$ However, the mechanism of action of miR-432-5p has not been previously investigated in CRC.

In the present study, the expression levels of circDUSP16 were assessed in CRC and the biological functions of circDUSP16 were investigated with regard to the progression of this disease. Moreover, the underlying molecular mechanism of the circDUSP16/miR-432$5 \mathrm{p} / \mathrm{E} 2 \mathrm{~F}$ transcription factor 6 (E2F6) axis was explored in vitro and in vivo.

\section{Materials and Methods}

\section{Clinical Samples}

A total of 46 CRC and adjacent normal tissues were collected from the Affiliated Changzhou No. 2 People's Hospital of Nanjing Medical University. All the samples were stored in liquid nitrogen. The present study was approved by the Ethics Committee of the Affiliated Changzhou No. 2 People's Hospital of Nanjing Medical University. Each patient provided written informed consent. All the experiments were performed in accordance with the Declaration of Helsinki.

\section{Cell Culture and Transfection}

The NCM460 colonic epithelial cell line was obtained from INCELL Corporation. The CRC cell lines SW480, HT-29, and HCT-116 were obtained from the Chinese Academy of Sciences. The cells were cultured in DMEM containing 10\% FBS (Gibco; Thermo Fisher Scientific, Inc.) and placed in a sterilized incubator with $5 \% \mathrm{CO}_{2}$ at $37^{\circ} \mathrm{C}$. Specific siRNA sequences were used against circDUSP16 or E2F6 (si-circDUSP16: 5'-ACCTGCTTG CAGGCTTTCAGT-3' or si-E2F6: 5'-UCAGUCACCCA AACGCAGCCA-3'), miRNA mimics or inhibitor targeting miR-432-5p (miR-432-5p mimics: 5'-ACGGUCA CCCAAACGCAGUUA-3' ormiR-432-5p inhibitor: 5'UAUGUGGAGACAACGCCCGAA-3'), the corresponding negative controls (NC mimic: 5'-CUCGGCCAUGUA GAGCGAAAA-3' or NC inhibitor: 5'-GGGUAUAUGA CCCAACCGGAA-3') and the siRNA scramble control (si-NC: 5'-GAUUCCAGACACACUGGAAGG $\left.-3^{\prime}\right)$. The pcDNA3.1-circDUSP16 overexpression vector (circDUSP16) and pcDNA3.1 empty vector (pcDNA) were purchased from GenePharma. Subsequently, Lipofectamine $^{\circledR} 2000$ (Invitrogen, Thermo Fisher Scientific, Inc.) was used to transfect si-circDUSP16 (40 $\mathrm{nM})$, si-E2F6 (40 nM), miR-432-5p mimics (40 nM), miR$432-5 \mathrm{p}$ inhibitor $(40 \mathrm{nM}), \mathrm{NC}$ mimic $(10 \mathrm{nM})$, NC inhibitor $(10 \mathrm{nM})$, si-NC $(10 \mathrm{nM})$, circDUSP16 $(40 \mathrm{nM})$ and pcDNA $(10 \mathrm{nM})$ into the cells.

\section{Reverse Transcription-Quantitative PCR (RT-qPCR)}

Total RNA was isolated from the tissues or cell lines with the TRIzol ${ }^{\circledR}$ reagent (Invitrogen; Thermo Fisher Scientific, Inc.) and reverse-transcribed into cDNA using PrimeScript RT Reagent kit (Takara Bio, Inc.). Subsequently, RT-qPCR was performed with the SYBR Premix Ex Taq II (Takara 
Bio, Inc.) in a 7500 Real-Time PCR system (Applied Biosystems; Thermo Fisher Scientific, Inc.). GAPDH or U6 were used as internal control genes. The relative expression levels were analyzed with the $2^{-\Delta \Delta \mathrm{Cq}}$ method. $^{22}$ The primer sequences used were as follows: circDUSP16 Forward (F): 5'-CCCAAGATGTTGCCTC TCTC-3' and Reverse (R): 5'-AGCCAGCGCATTACA TCATT-3'; E2F6 (F): 5'-TGTGCTCAGCAGCTGTTTG AGT-3' and (R): 5'-TGTTCATGGAAGGCCTGAATG-3'; miR-432-5p (F): 5'-AACGAGACGACGACAGAC-3' and (R): 5'-CTTGGAGTAGGTCATTGGGT-3'; GAPDH (F): 5'-TGCACCACCAACTGCTTAGC-3' and (R): 5'-GGCA TGGACTGTGGTCATGAG-3'; and U6 (F): 5'-AACGAG ACGACGACAGAC-3' and (R): 5'-GCAAATTCGTGA AGCGTTCCATA-3'.

\section{CCK-8 Assay}

The CCK-8 assay (Dojindo Molecular Technologies, Inc.) was performed to detect cell viability following the manufacturer's protocol. Briefly, the cells were seeded in 96well plates and incubated for $0,24,48$, and $72 \mathrm{~h}$. Subsequently, the CCK-8 solution was added to each well and the cells were incubated for $2 \mathrm{~h}$. The absorbance was determined with a microplate reader (Bio-Rad Laboratories, Inc.) at $450 \mathrm{~nm}$.

\section{Transwell Assay}

The cell migratory and invasive activities were analyzed in Transwell insert chambers (24-well, $8-\mu \mathrm{m}$ pore size; Corning Inc.). The invasion assay was performed by seeding the cells into the upper chamber with a serum-free medium. The lower surface of the upper chamber was coated with Matrigel. Subsequently, a growth medium containing $10 \%$ FBS was placed in the lower chamber. The cells were allowed to proliferate for $24 \mathrm{~h}$ and the invading cells were fixed and stained with crystal violet. The number of the cells was counted by a light microscope (Olympus Corporation; x200). Matrigel was not used in the migration assay; the other steps were performed in the same manner.

\section{Flow Cytometry}

In the present study, the apoptosis of CRC cells was assessed with the Annexin V-FITC Apoptosis Detection Kit (Beyotime Institute of Biotechnology). The analysis was performed using flow cytometry. Briefly, transfected SW480 and HT-29 cells were collected and washed with ice-cold PBS following $48 \mathrm{~h}$ of cell culture. The cells were collected and resuspended in PBS at $4{ }^{\circ} \mathrm{C}$. A total of $5 \mu \mathrm{L}$ Annexin V-FITC and $5 \mu \mathrm{L}$ PI were added to the cell suspension. Following incubation at $25^{\circ} \mathrm{C}$ in the dark for 15 min, the number of apoptotic cells was assessed with a flow cytometer (BD Biosciences).

\section{Western Blot Analysis}

Western blotting was performed following standard methodologies. Initially, the total protein was extracted and quantified using a BCA kit (Thermo Fisher Scientific, Inc.). The protein samples were transferred on PVDF membranes (Pall Corporation) and separated using 10\% SDS-PAGE (Beijing Solarbio Science \& Technology Co., Ltd.). Subsequently, the blots were blocked in $10 \%$ non-fat milk for $2 \mathrm{~h}$ and probed with primary antibodies against $\mathrm{Bcl}-2$ (cat. no. ab196495; 1:1,000; Abcam), Bax (cat. no. ab182733; 1:2,000; Abcam), cleaved-caspase 3 (cat. no. ab32042; 1:1,000; Abcam) or GAPDH (cat. no. ab181602; 1:5,000; Abcam) overnight at $4{ }^{\circ} \mathrm{C}$. The proteins were probed with HRP-conjugated secondary antibody (cat. no. D110150; 1:5,000; Sangon Biotech Co., Ltd.) for $2 \mathrm{~h}$. The protein bands were analyzed with an enhanced chemiluminescence reagent (Beyotime Institute of Biotechnology).

\section{Xenograft Model}

The animal experiments were carried out in accordance with the National Guidelines for the Experimental Animal Welfare and approved by the Ethics Committee of the Affiliated Changzhou No. 2 People's Hospital of Nanjing Medical University. To establish the CRC murine xenograft model, 4-week-old BALB/c nude mice were purchased from Charles River Laboratories. HT-29 cells $\left(2 \times 10^{6}\right)$, transfected with si-NC or si-circDUSP16 (stable transfectants), were subcutaneously injected into the right abdomen of the nude mice. The tumors were allowed to grow for 28 days and the mice were sacrificed by cervical dislocation following deep anesthesia with sodium pentobarbital $(50 \mathrm{mg} / \mathrm{kg})$. The tumors were then resected and weighed. The tumor volume was calculated with the following equation: Volume $=\left(\right.$ length $\mathrm{x}$ width $\left.^{2}\right) / 2$ and the maximum tumor volume was $986 \mathrm{~mm}^{3}$.

\section{Bioinformatics Analysis}

StarBase database (http://starbase.sysu.edu.cn/) was used to predict interactions between miR-432-5p and circDUSP16 or E2F6 by searching for the binding sites. 


\section{Luciferase Reporter Assay}

CircDUSP16 or E2F6 sequences containing wild-type (WT) or mutant (MUT) binding sites for miR-432-5p were synthesized and cloned into the pGL3 reporter vector (Promega Corporation) to construct Luciferase reporter vectors for the Luciferase reporter assay. The Dual-Luciferase Reporter Assay System (Promega Corporation) was used to measure Luciferase activities of the reporter vectors following the manufacturer's instructions.

\section{RNA-Binding Protein}

\section{Immunoprecipitation (RIP) Assay}

Rip assay was performed with EZ-Magna RIP Kit (Millipore, USA). In brief, the cells were lysed in RIP lysis buffer and then incubated in RIP buffer containing magnetic beads conjugated with negative control (normal mouse $\mathrm{IgG}$ ) or human anti-Argonaute 2 (Ago2, Millipore). Thereafter, the coprecipitated RNA was isolated, purified, and finally subjected to RT-qPCR.

\section{Statistical Analysis}

The GraphPad Prism 6 (GraphPad Software, Inc.) was used for statistical analysis and the data are expressed as mean \pm standard deviation of at least three independent experimental repeats. The paired/unpaired Student's $t$-test or one-way ANOVA test, followed by Tukey's post hoc test was used to compare two and multiple groups, respectively. The overall survival of patients with CRC was analyzed by the Kaplan-Meier method and the Log rank test. The cut-off point was determined using the mean value of circDUSP16 or E2F6 expression. Patients with circDUSP 16 or E2F6 expression above or below the mean value were divided into the high expression group and the low expression group, respectively. All the experiments were performed in triplicate. $\mathrm{P}<0.05$ was considered to indicate statistically significant differences.

\section{Results \\ CircDUSPI6 Expression is Upregulated in CRC}

CircDUSP16 expression was examined in CRC and adjacent normal tissues and the results indicated that it was upregulated in CRC tissues compared to the corresponding expression noted in adjacent normal tissues (Figure 1A). Moreover, clinical data showed that high expression of circDUSP16 was associated with tumor size and TNM stage (Table 1). In addition, circDUSP16 expression in CRC cell lines (SW480, HT-29, and HCT-116) and the colonic epithelial cell line (NCM460) was also detected. It was shown that circDUSP16 levels were notably increased in CRC cell lines (Figure 1B). Subsequently, Kaplan-Meier analysis indicated that CRC patients with high circDUSP16 expression exhibited poorer prognosis compared with patients with low circDUSP16 expression in our patient cohort (Figure 1C). Therefore, circDUSP16 may facilitate CRC progression.

\section{CircDUSPI6 Knockdown Inhibits Proliferation and Metastasis and Induces Apoptosis of CRC Cells}

Since circDUSP16 expression was higher in SW480 and HT-29 cells, the cells were selected for subsequent investigations. CircDUSP16 expression was knocked down in SW480 and HT-29 cells (Figure 2A). CircDUSP16 knockdown inhibited cell proliferation as determined by the CCK-8 assay (Figure 2B). To investigate the function of circDUSP16 in CRC metastasis, the Transwell assay was performed. The data indicated that circDUSP16
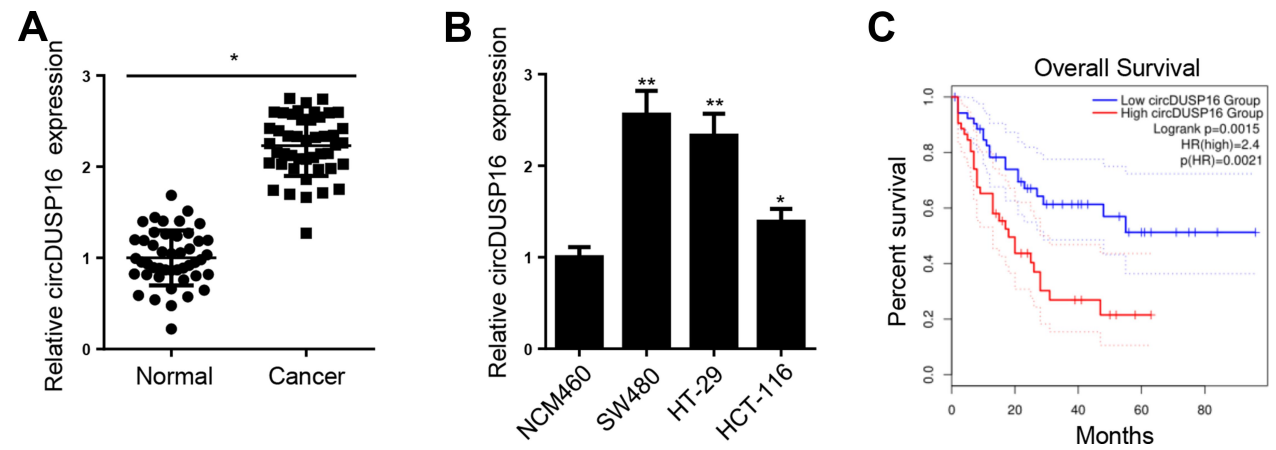

Figure I CircDUSPI6 is upregulated in CRC. (A) CircDUSPI6 expression in 28 CRC tissues ( $\mathrm{n}=46$ ) and their adjacent normal controls ( $\mathrm{n}=46$ ) was measured. (B) CircDUSPI6 level was upregulated in CRC cell lines. (C) Kaplan-Meier analysis was conducted to illustrate the correlation between circDUSPI6 expression and patients' survival. ${ }^{*} \mathrm{P}<0.05, * * \mathrm{P}<0.01$. 
Table I The Correlation Between circDUSPI6 Expression and Clinicopathological Features in CRC Patients

\begin{tabular}{|l|c|c|c|c|}
\hline \multirow{2}{*}{ Variables } & \multirow{2}{*}{ Number } & \multicolumn{2}{|c|}{ circDUSP I6 } & \multirow{2}{*}{ P value } \\
\cline { 3 - 4 } & & $\begin{array}{c}\text { Low } \\
(\mathbf{n}=\mathbf{2 2})\end{array}$ & $\begin{array}{c}\text { High } \\
(\mathbf{n}=\mathbf{2 4})\end{array}$ & \\
\hline $\begin{array}{c}\text { Age } \\
<60\end{array}$ & 13 & 13 & 10 & 0.183 \\
$\geq 60$ & 23 & 9 & 14 & \\
\hline $\begin{array}{c}\text { Gender } \\
\text { Male }\end{array}$ & 30 & 14 & 16 & 0.874 \\
Female & 16 & 8 & 8 & \\
\hline $\begin{array}{c}\text { Tumor size } \\
<5\end{array}$ & 20 & 16 & 4 & $0.018^{*}$ \\
$\geq 5$ & 26 & 6 & 20 & \\
\hline $\begin{array}{c}\text { TNM stage } \\
\text { I+II }\end{array}$ & 25 & 18 & 7 & $0.013^{*}$ \\
III+IV & 21 & 4 & 17 & \\
\hline
\end{tabular}

Note: $* \mathrm{P}<0.05$.

downregulation repressed migration and invasion of CRC cells (Figure 2C and D). Flow cytometry analysis indicated that circDUSP16 deletion contributed to increased apoptosis of SW480 and HT-29 cells compared to the corresponding levels noted in the control group (Figure 2E). In addition, Western blot analysis was performed to evaluate the expression levels of the proapoptotic protein (Bax and Cleaved-Caspase 3) and the anti-apoptotic protein Bcl-2 in SW480 and HT-29 cells. The results indicated that circDUSP16 knockdown markedly upregulated $\mathrm{Bax}$ and cleaved-caspase 3 expression, whereas it caused a significant downregulation in Bcl-2 expression compared to the corresponding levels noted in the control group (Figure 2F), indicating that circDUSP16 was implicated in CRC cell apoptosis. Therefore, circDUSP16 may act as an oncogenic circRNA in CRC.

\section{CircDUSPI6 Deficiency Impairs Malignant Properties of CRC Cells as a Sponge for miR-432-5p}

To explore the detailed circDUSP16-regulated network in CRC, firstly, bioinformatics analysis was carried out to find the downstream targets of circDUSP16. StarBase website predicted miR-432-5p as a target of circDUSP16, with latent binding sites as shown in Figure 3A. Subsequently, the transfection efficiency for miR-432-5p mimics and inhibitor was also confirmed (Figure 3B).
Luciferase reporter assays indicated that miR-432-5p mimics repressed the activity of the circDUSP16-WT reporter, whereas the miR-432-5p inhibitor stimulated the activity of the circDUSP16-WT reporter; however, no change was noted in the activity of the circDUSP16MUT reporter (Figure 3C). RIP assay also demonstrated that circDUSP16 and miR-432-5p were preferentially enriched in anti-Ago2 group (Figure 3D). In addition, circDUSP16 knockdown caused an upregulation in miR432-5p expression, whereas circDUSP16 overexpression downregulated miR-432-5p expression levels in CRC cells (Figure 3E). All these results demonstrated that circDUSP16 negatively regulates miR-432-5p in CRC cells. To further verify whether miR-432-5p participated in the circDUSP16-regulated CRC progression, HT-29 and SW480 cells were respectively transfected with si-NC, sicircDUSP16, si-circDUSP16 $+\mathrm{NC}$ inhibitor, or sicircDUSP16 + miR-432-5p inhibitor. It was discovered that miR-432-5p inhibition abrogated the effects of circDUSP16 blocking on CRC cell proliferation (Figure 3F), migration and invasion (Figure $3 \mathrm{G}$ and $\mathrm{H}$ ), as well as apoptosis (Figure 3I). To sum up, circDUSP16 depletion restrains CRC malignant processes in vitro via inversely regulating miR-432-5p.

\section{E2F6 is a Direct Target of miR-432-5p}

E2F6 was predicted as a target of miR-432-5p by StarBase website (Figure 4A). Moreover, TCGA database indicated that E2F6 expression was highly expressed in COAD (Figure 4B). To confirm this prediction, luciferase reporter assay was performed. The results indicated that transfection with miR-432-5p mimics markedly suppressed E2F6-WT reporter activity, whereas transfection with the miR-432-5p inhibitor promoted the E2F6-WT reporter activity. However, the E2F6-MUT reporter activity did not exhibit profound changes (Figure 4C). As indicated by RIP assay, miR-432-5p and E2F6 were preferentially enriched in antiAgo2 group (Figure 4D). Moreover, miR-432-5p mimics downregulated E2F6 expression, while the miR-432-5p inhibitor induced its upregulation (Figure 4E). Therefore, the present study indicated that miR-432-5p negatively modulated E2F6 expression in CRC cells.

\section{CircDUSPI6 Promotes CRC Progression} via the miR-432-5p/E2F6 Axis in vitro

RT-qPCR assay indicated that E2F6 expression was upregulated in CRC tissues (Figure 5A), suggesting that E2F6 

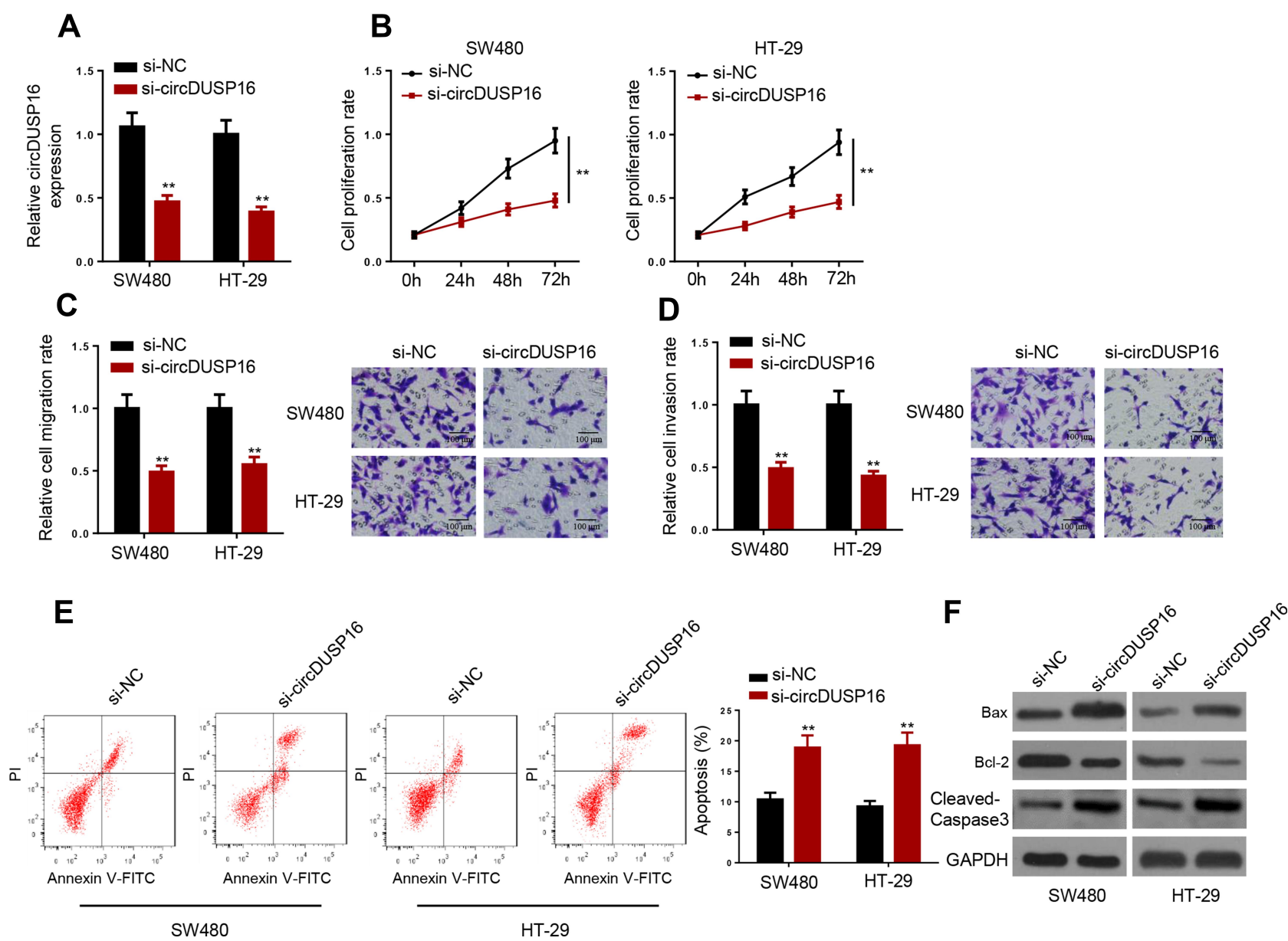

Figure 2 CircDUSPI6 knockdown inhibits proliferation and metastasis and induces apoptosis of CRC cells. (A) Expression detection of circDUSPI6 in SW480 and HT-29 cells. (B) CCK-8 assay was carried out to test the effects of circDUSPI6 knockdown on proliferation. (C and D) Transwell assay was conducted to analyze migration and invasion. (E) Flow cytometry analysis showed the apoptosis rate of CRC cells transfected with si-NC and si-circDUSPI6. (F) Western blot indicated the protein levels of Bax, Cleaved-Caspase 3, and $\mathrm{Bcl}-2$. $* * \mathrm{P}<0.0 \mathrm{l}$.

is a potential oncogene involved in CRC progression. Subsequently, Kaplan-Meier analysis indicated high E2F6 expression was correlated with low survival rate (Figure 5B). It was found that E2F6 knockdown partially reversed the effect of the miR-432-5p inhibitor on proliferation, migration, and invasion of CRC cells (Figure 5CE). Moreover, the results of the flow cytometry analysis suggested that E2F6 inhibition partly abolished the suppressive effect on CRC cell apoptosis induced by downregulation of miR-432-5p expression (Figure 5F). In addition, E2F6 depletion partially reversed the effects on Bax, cleaved-caspase 3, and Bcl-2 expression levels caused by miR-432-5p inhibition (Figure 5G). Furthermore, circDUSP16 knockdown suppressed E2F6 expression, and such an impact was abolished by treating the cells with miR-432-5p inhibitor (Figure 5H), indicating that circDUSP 16 positively regulated E2F6 expression by interacting with miR-432-5p. Collectively, the results indicated that circDUSP16 may contribute to CRC progression by regulating the miR-432-5p/E2F6 axis in vitro.

\section{Loss of circDUSPI6 Impairs CRC Growth in vivo}

To verify the effects of circDUSP16 on tumor growth in vivo, HT-29 cells were transfected with si-NC or sicircDUSP16 (stable transfectants) and injected into nude mice. The tumor volume and weight were apparently decreased following circDUSP16 depletion compared to the corresponding effects noted in the control group (Figure 6A and B). Moreover, the RT-qPCR assay was also performed to detect circDUSP16, miR-432-5p, and E2F6 expression levels in the tumor models. The results demonstrated that the expression levels of circDUSP 16 and E2F6 were downregulated, while those of miR-432$5 \mathrm{p}$ were upregulated in the tumor model (circDUSP16 knockdown cells; Figure 6C). These data confirmed that 

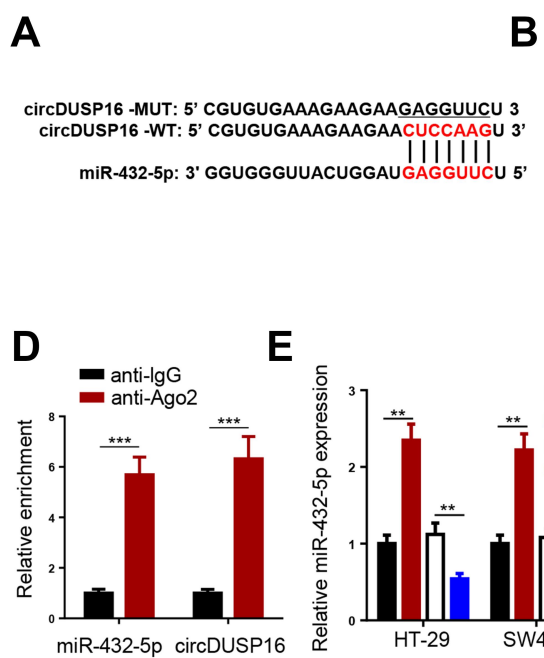

G
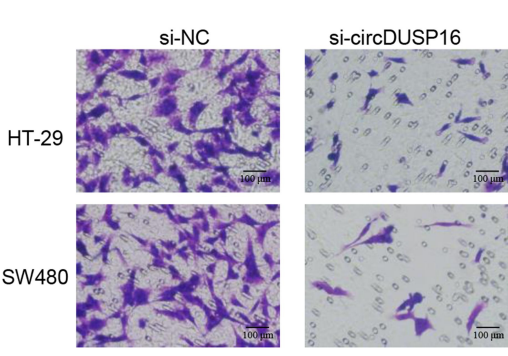

H
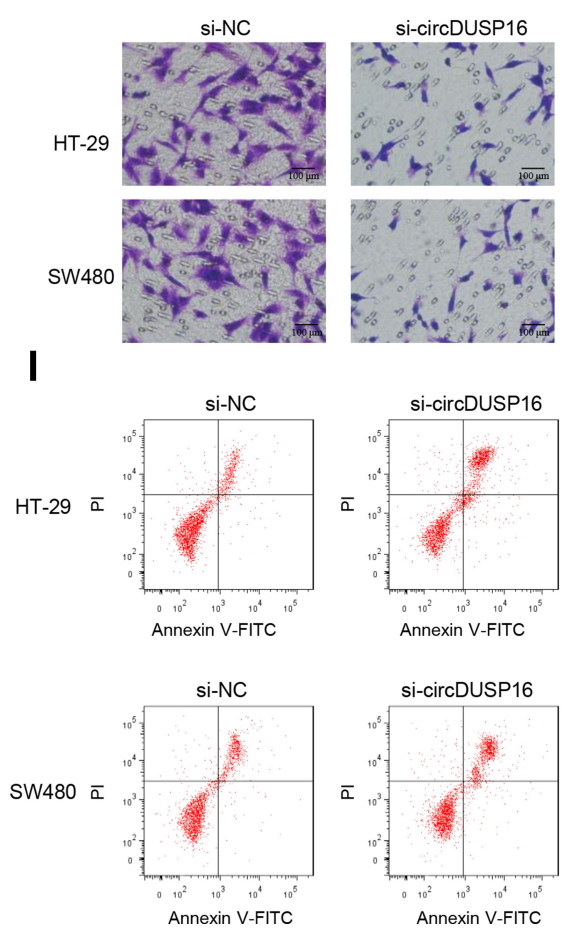

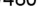

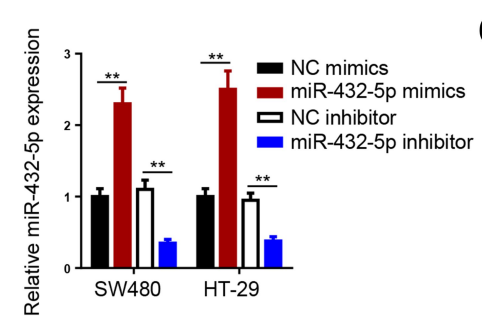

F $\quad \mathrm{HT}-29$

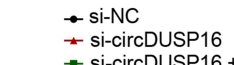

- si-circDUSP16 + NC inhibito

(2) 1.5 - si-circDUSP16 +
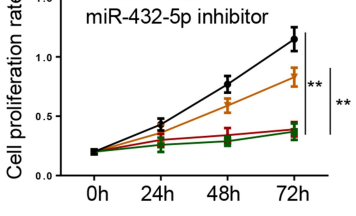

C

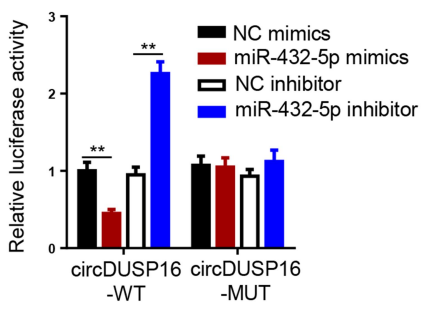

SW480

$\rightarrow$ si-NC

- si-circDUSP16

- si-circDUSP16 + NC inhibitor

\& 1.57 - si-circDUSP16 +

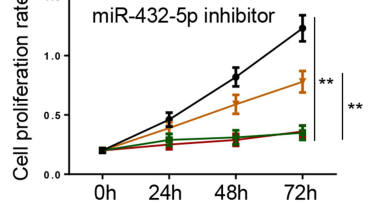

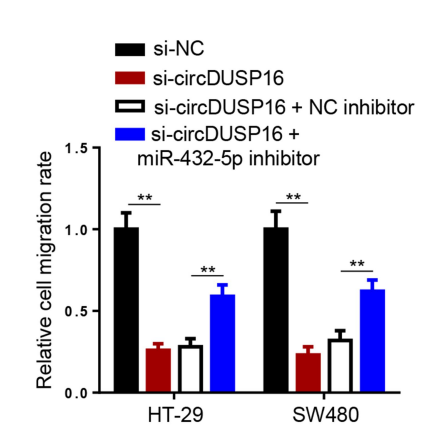
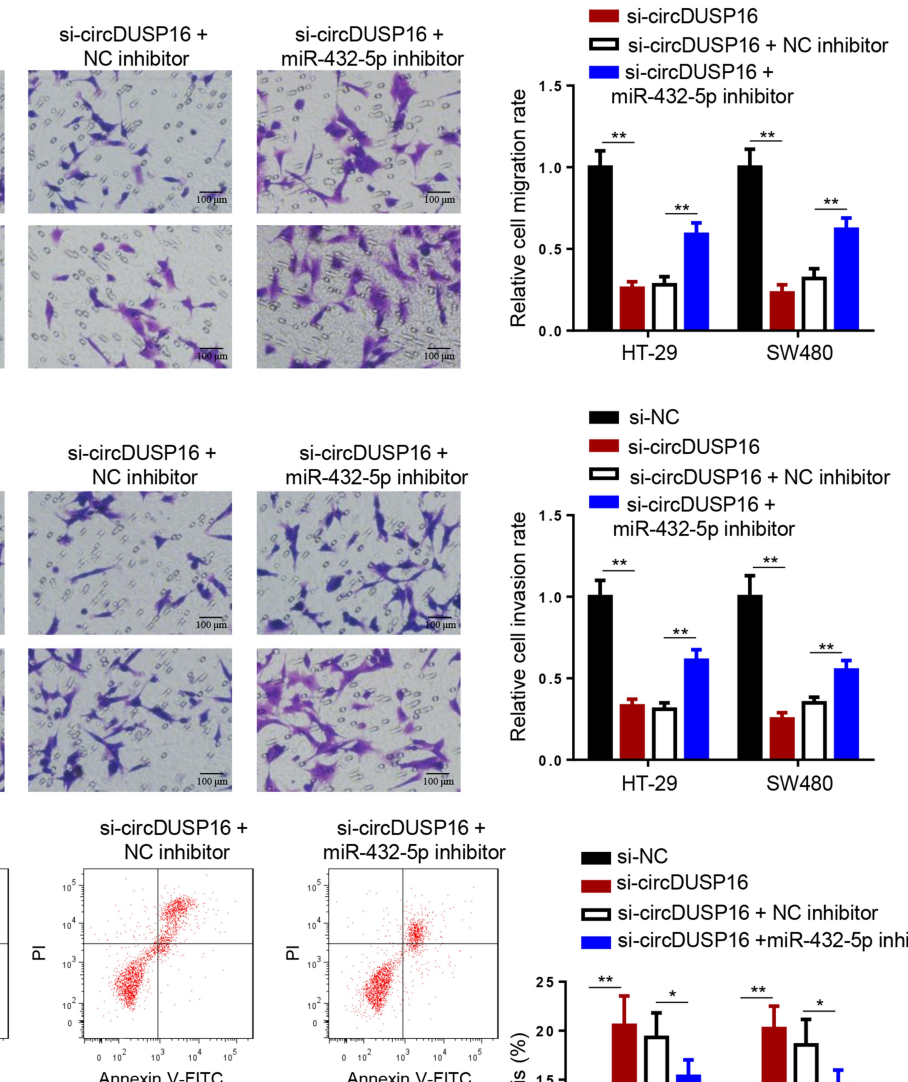

si-circDUSP16+
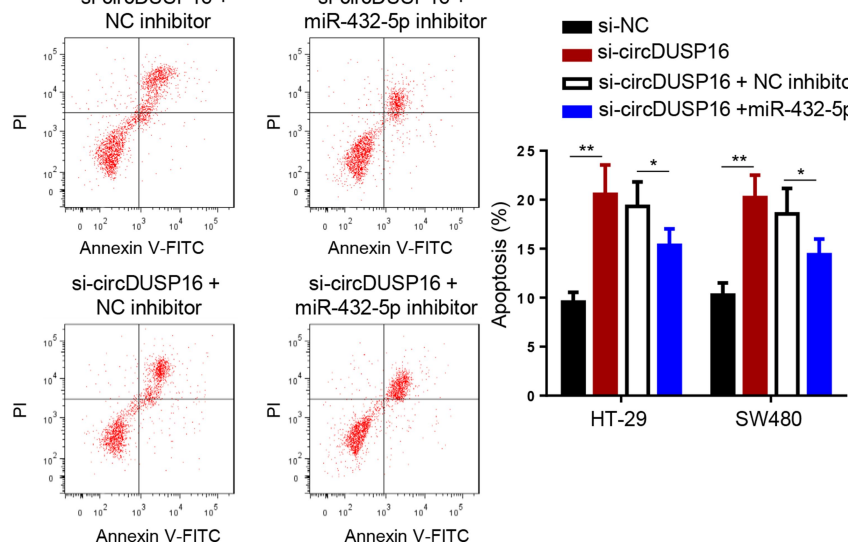

Figure 3 CircDUSPI6 deficiency impairs malignant properties of CRC cells as a sponge for miR-432-5p. (A) StarBase website predicted the complementary sequence between circDUSPI6 and miR-432-5p. (B) Expression analysis of miR-432-5p after transfection with miR-432-5p mimics, miR-432-5p inhibitor, or negative controls. (C) Luciferase reporter assay was performed to analyze the activity of circDUSPI6-WT or circDUSPI6-MUT reporter in SW480 cells. (D) RIP assay demonstrated that circDUSPI6 and miR-432-5p co-existed in RISC. (E) miR-432-5p expression was analyzed after transfection with indicated vectors. (F) Cell proliferation was analyzed by CCK-8 assay. (G and $\mathbf{H}$ ) Transwell assay was used to detect cell migration and invasion abilities. (I) Flow cytometry analysis was applied to analyze cell apoptosis. $*$ P $<0.05$, $* * \mathrm{P}<0.01$, **** $\mathrm{P}<0.001$. 

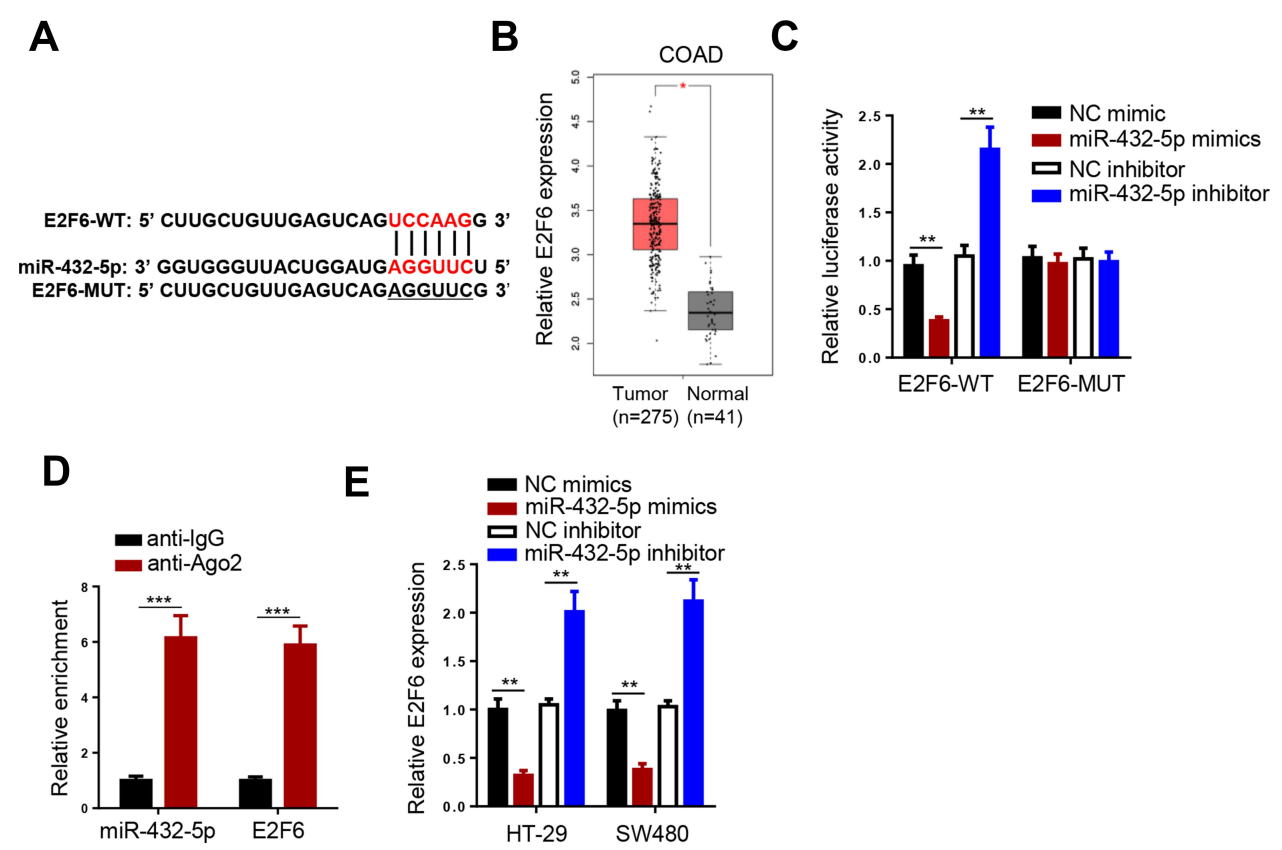

Figure 4 E2F6 is a direct target of miR-432-5p. (A) StarBase website predicted the complementary sequence between E2F6-3'UTR and miR-432-5p. (B) ATCG database showed the expression of E2F6 in COAD. (C) Luciferase reporter assay was performed to analyze the activity of E2F6-WT or E2F6-MUT reporter in SW480 cells. (D) RIP assay demonstrated that miR-432-5p and E2F6 co-existed in RISC. (E) E2F6 expression was inhibited by miR-432-5p. $* * \mathrm{P}<0.0 \mathrm{I}$, $* * * \mathrm{P}<0.00 \mathrm{I}$.

circDUSP16 inhibition inhibited CRC growth by regulating miR-432-5p and E2F6 levels in vivo.

\section{Discussion}

Numerous studies have demonstrated that circRNAs serve as important regulators of CRC progression by stimulating various biological behaviors. For example, Zhou et al found that circRNA CAMSAP1 facilitated CRC tumor growth by upregulating E2F1 as a sponge of miR-328$5 \mathrm{p} .{ }^{23}$ As reported by Shang et al, exosome-induced circPACRGL contributed to CRC tumorigenesis by regulating miR-142-3p/miR-506-3p-TGF- $\beta 1$ axis. ${ }^{24}$ Besides, Dai et al also revealed that Circ-UBAP2 promotes CRC progression via targeting the miR-199a/VEGFA axis. ${ }^{25}$ These results have demonstrated the important function of circRNAs in regulating CRC progression. In the present study, the correlation between circDUSP16 expression and CRC progression was examined and the results indicated that circDUSP16 was highly expressed in CRC tissues and cell lines. In addition, the data suggested that high circDUSP16 expression was correlated with low survival rate of patients with CRC. By using functional experiments, it was demonstrated that circDUSP16 knockdown repressed the proliferation, migration, and invasion of CRC cells, and induced CRC cell apoptosis. This was achieved by increasing the expression levels of Bax and reducing the expression levels of $\mathrm{Bcl}-2$. Moreover, circDUSP16 silencing inhibited CRC tumor growth in vivo. Therefore, the findings identified circDUSP16 as a novel oncogenic circRNA involved in CRC progression.

It has been widely recognized that several circRNAs may interact with specific miRNAs to exert their effects on various aspects of cancer development. ${ }^{26}$ In the present study, miR-432-5p was identified as a potential miRNA of circDUSP16 using bioinformatic databases. A limited number of studies have examined the contribution of miR-432-5p in cancer development. Xu et al demonstrated that high miR-432-5p expression repressed pancreatic carcinoma progression by targeting PPME $1 .{ }^{27}$ Liu et al discovered that decreased miR-432-5p expression may facilitate the proliferation and migration of breast cancer cells by modulating E2F3 expression. ${ }^{18}$ In addition, Liu et al demonstrated that miR-432-5p was also involved in the proliferation and metastasis of hepatocellular carcinoma cells. ${ }^{19}$ In the present study, the direct interaction between circDUSP16 and miR-432-5p was assessed using Luciferase reporter and RIP assays. The data indicated that circDUSP16 repressed miR-432-5p levels, suggesting that the latter served as a tumor suppressor in CRC. A series of functional assays

Subsequently, the downstream target of miR-432-5p was also analyzed. E2F6 was predicted and confirmed as 
A

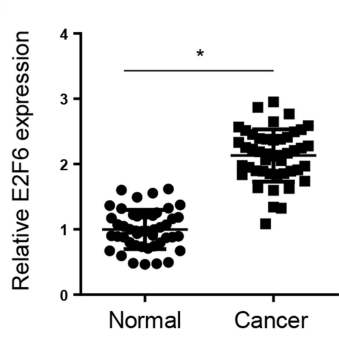

B

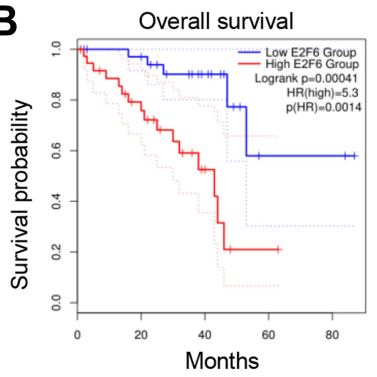

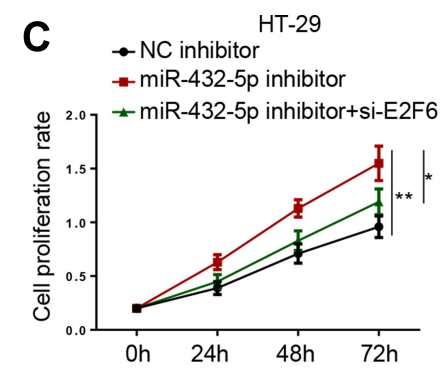

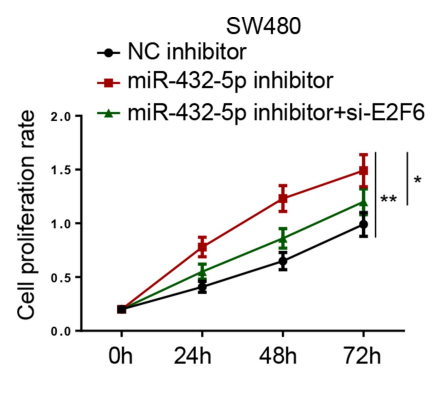

SW480

D

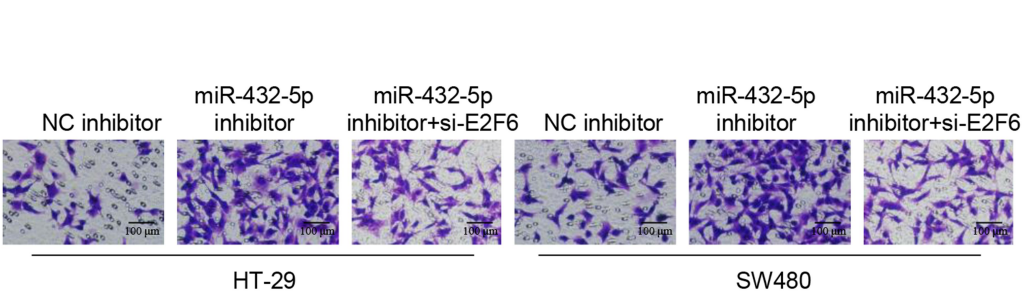

E
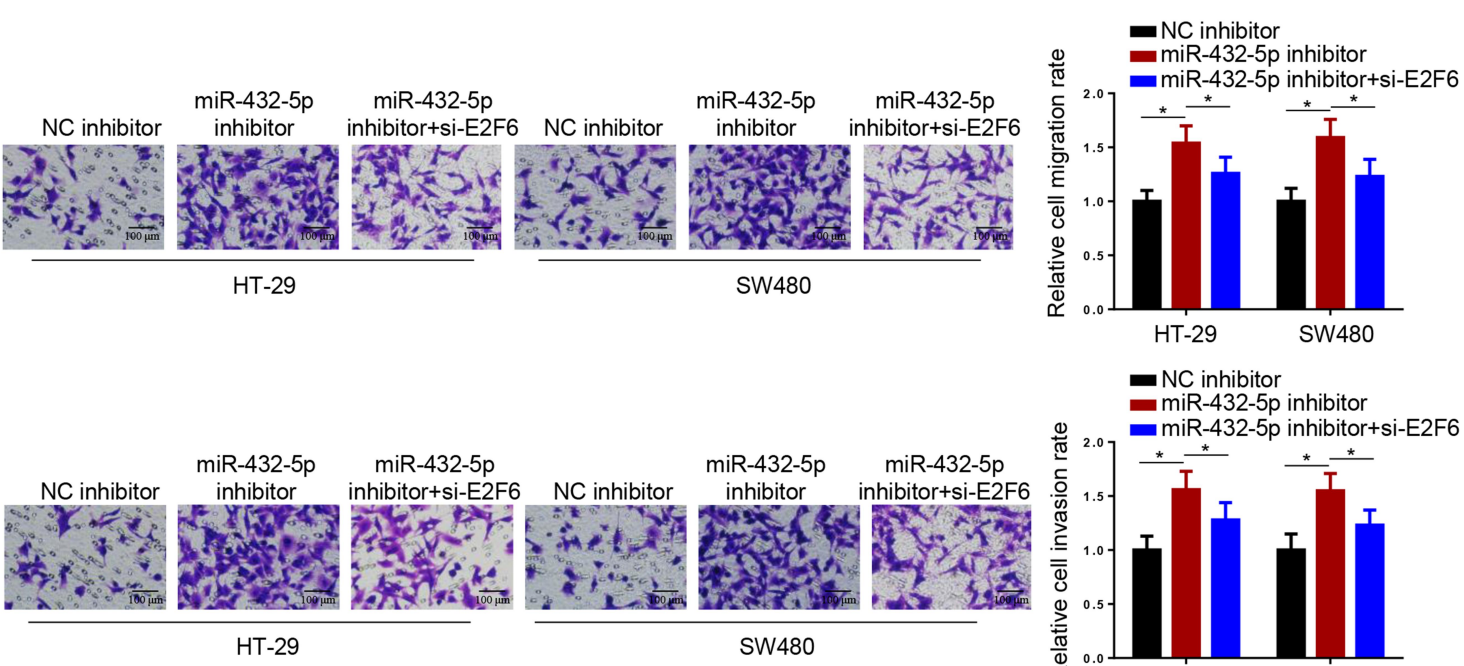

F

HT-29

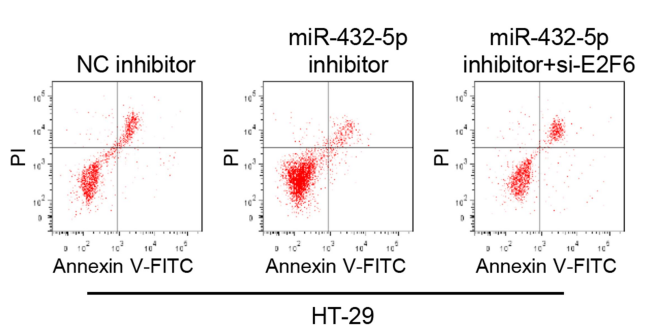

HT-29

SW480

- NC inhibitor

- miR-432-5p inhibitor
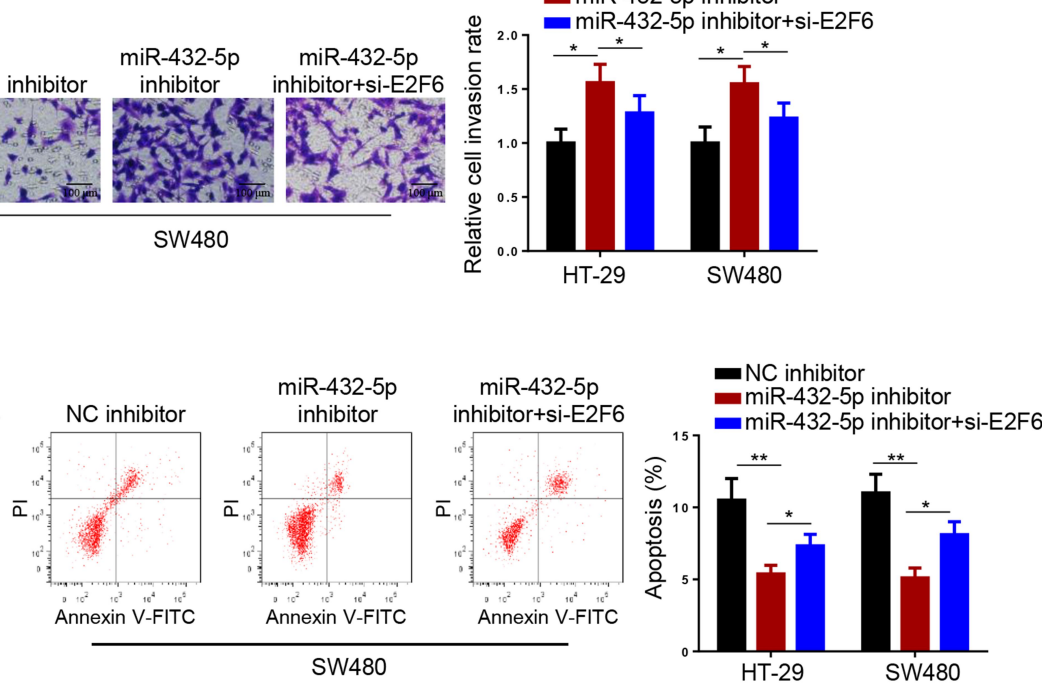

G

H
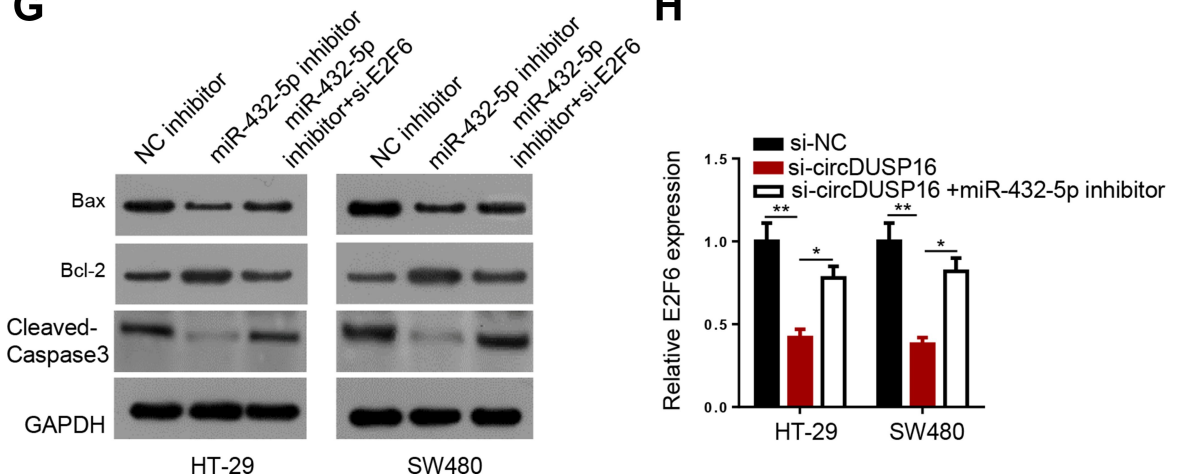

Figure 5 CircDUSPI 6 promotes CRC progression through miR-432-5p-3p/E2F6 axis in vitro. (A) Relative E2F6 expression in CRC tissues ( $n=46)$. (B) Kaplan-Meier analysis was conducted to illustrate the correlation between E2F6 expression and patients' survival. (C) CCK-8 assay was performed after transfection with indicated plasmids. (D and E) Transwell assay was conducted to analyze migration and invasion. (F) Flow cytometry analysis showed the apoptosis rate of CRC. (G) Western blot indicated the protein levels of Bax, Cleaved-Caspase 3, and Bcl-2. (H) RT-qPCR showed the expression of E2F6 in CRC cells transfected with si-NC, si-circDUSPI6, and si-circDUSPI6 + miR-432-5P inhibitor. *P<0.05, **P<0.01. 

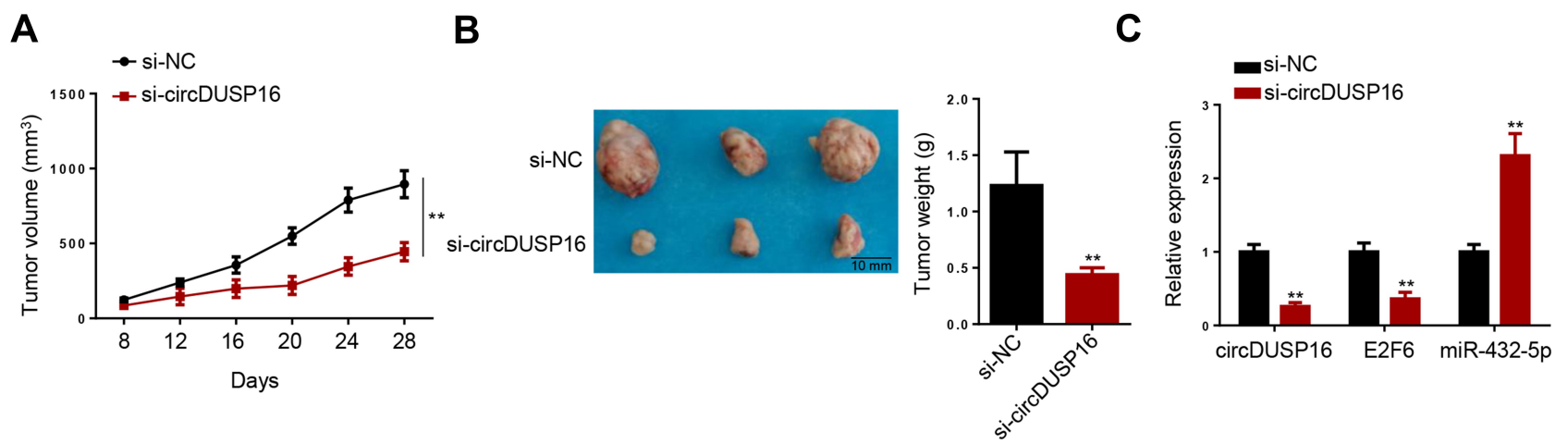

Figure 6 Loss of circDUSPI6 impairs CRC tumor growth in vivo. (A) the tumor growth curves were plotted according to the tumor volumes measured every 4 days. (B) Tumor weight was examined after cells were injected for 28 days. (C) circDUSPI6, miR-432-5p, and E2F6 expressions were determined by RT-qPCR assay. $* *$ P $<0.01$.

a target for miR-432-5p. A large number of studies have demonstrated that E2F6 is an oncogene involved in the progression of several types of cancer. Suppression of E2F6 expression inhibits the development of renal cell carcinoma. ${ }^{28}$ E2F6 has been shown to promote cell proliferation. ${ }^{29}$ Cheng et al indicated that E2F6 acted as an oncogene to promote ovarian cancer stemness. ${ }^{30}$ However, its function in CRC has not been previously studied. It was herein demonstrated that E2F6 levels were increased in CRC tissues, whereas E2F6 expression was upregulated by circDUSP16 via its interaction with miR-432-5p. In addition, the results of the functional experiments indicated that E2F6 knockdown partially repressed the accelerated CRC cell growth and metastasis, and it also suppressed apoptosis induced by miR-432-5p inhibition. Therefore, the present study demonstrated that circDUSP16 may regulate CRC progression via the miR-432-5p/E2F6 axis.

In conclusion, the results indicated that the circDUSP16/miR-432-5p/E2F6 regulatory network was involved in CRC progression. Downregulation of circDUSP16 expression resulted in higher miR-432-5p and lower E2F6 expression levels. These data suggest that the circDUSP16/miR-432-5p/E2F6 axis may represent a promising target for $\mathrm{CRC}$ treatment.

\section{Disclosure}

The authors report no conflicts of interest for this work.

\section{References}

1. Ferlay J, Soerjomataram I, Dikshit R, et al. Cancer incidence and mortality worldwide: sources, methods and major patterns in GLOBOCAN 2012. Int $J$ Cancer. 2015;136:E359-E386. doi:10.10 02/ijc. 29210
2. Day LW, Velayos F. Colorectal cancer screening and surveillance in the elderly: updates and controversies. Gut Liver. 2015;9:143-151. doi: $10.5009 / \mathrm{gnl} 14302$

3. Siegel RL, Miller KD, Fedewa SA, et al. Colorectal cancer statistics, 2017. CA Cancer J Clin. 2017;67:177-193.

4. Araghi M, Soerjomataram I, Jenkins M, et al. Global trends in colorectal cancer mortality: projections to the year 2035. Int $J$ Cancer. 2019;144:2992-3000. doi:10.1002/ijc.32055

5. Harris TJ, McCormick F. The molecular pathology of cancer. Nat Rev Clin Oncol. 2010;7:251-265. doi:10.1038/nrclinonc.2010.41

6. Weng W, Wei Q, Toden S, et al. Circular RNA ciRS-7-A promising prognostic biomarker and a potential therapeutic target in colorectal cancer. Clin Cancer Res. 2017;23:3918-3928. doi:10.1158/10780432.CCR-16-2541

7. Chen J, Li Y, Zheng Q, et al. Circular RNA profile identifies circPVT1 as a proliferative factor and prognostic marker in gastric cancer. Cancer Lett. 2017;388:208-219. doi:10.1016/j.canlet.2016.12.006

8. Chen D, Ma W, Ke Z, Xie F. CircRNA hsa_circ_100395 regulates miR-1228/TCF21 pathway to inhibit lung cancer progression. Cell Cycle. 2018;17:2080-2090. doi:10.1080/15384101.2018.1515553

9. Wong CH, Lou UK, Li Y, et al. CircFOXK2 promotes growth and metastasis of pancreatic ductal adenocarcinoma by complexing with RNA-binding proteins and sponging MiR-942. Cancer Res. 2020;80:2138-2149. doi:10.1158/0008-5472.CAN-19-3268

10. Zeng K, Chen X, Xu M, et al. CircHIPK3 promotes colorectal cancer growth and metastasis by sponging miR-7. Cell Death Dis. 2018;9:417. doi:10.1038/s41419-018-0454-8

11. Zhang Z, Wang C, Zhang Y, Yu S, Zhao G, Xu J. CircDUSP16 promotes the tumorigenesis and invasion of gastric cancer by sponging miR-145-5p. Gastric Cancer. 2020;23:437-448. doi:10.1007/ s10120-019-01018-7

12. Ma L, Li H, Lin Y, et al. CircDUSP16 contributes to cell development in esophageal squamous cell carcinoma by regulating miR-4975p/TKTL1 axis. J Surg Res. 2021;260:64-75. doi:10.1016/j. jss.2020.11.052

13. Tian $\mathrm{Y}, \mathrm{Xu} \mathrm{Y}$, Wang $\mathrm{H}$, et al. Comprehensive analysis of microarray expression profiles of circRNAs and IncRNAs with associated co-expression networks in human colorectal cancer. Funct Integr Genomics. 2019;19:311-327. doi:10.1007/s10142-018-0641-9

14. Bartel DP. MicroRNAs: genomics, biogenesis, mechanism, and function. Cell. 2004;116:281-297. doi:10.1016/S0092-8674(04)00045-5

15. Wen D, Li S, Jiang W, Zhu J, Liu J, Zhao S. miR-539 inhibits human colorectal cancer progression by targeting RUNX2. Biomed Pharmacother. 2017;95:1314-1320. doi:10.1016/j.biopha.2017.09. 044

16. Zhang W, Chen X, Jia J. MiR-3150b-3p inhibits the progression of colorectal cancer cells via targeting GOLPH3. J Investig Med. 2020;68:425-429. doi:10.1136/jim-2019-001124 
17. Zhu QD, Zhou QQ, Dong L, Huang Z, Wu F, Deng X. MiR-199a-5p inhibits the growth and metastasis of colorectal cancer cells by targeting ROCK1. Technol Cancer Res Treat. 2018;17:1533034 618775509. doi:10.1177/1533034618775509

18. Liu Y, Lu C, Zhou Y, Zhang Z, Sun L. Circular RNA hsa_circ 0008039 promotes breast cancer cell proliferation and migration by regulating miR-432-5p/E2F3 axis. Biochem Biophys Res Commun. 2018;502:358-363. doi:10.1016/j.bbrc.2018.05.166

19. Liu H, Xue L, Song C, Liu F, Jiang T, Yang X. Overexpression of circular RNA circ_001569 indicates poor prognosis in hepatocellular carcinoma and promotes cell growth and metastasis by sponging miR-411-5p and miR-432-5p. Biochem Biophys Res Commun. 2018;503:2659-2665. doi:10.1016/j.bbrc.2018.08. 020

20. Xiong X, Feng J, Yang X, Li H, Shi Q, Tao J. Circular RNA CDR1as promotes tumor progression by regulating miR-432-5p/E2F3 axis in pancreatic cancer. Cancer Cell Int. 2021;21:1-10. doi:10.1186/s12 935-021-01812-3

21. Luo M, Hu Z, Kong Y, Li L. MicroRNA-432-5p inhibits cell migration and invasion by targeting CXCL5 in colorectal cancer. Exp Ther Med. 2021;21:301. doi:10.3892/etm.2021.9732

22. Livak KJ, Schmittgen TD. Analysis of relative gene expression data using real-time quantitative PCR and the $2-\Delta \Delta C T$ method. Methods. 2001;25:402-408. doi:10.1006/meth.2001.1262

23. Zhou C, Liu HS, Wang FW, et al. circCAMSAP1 promotes tumor growth in colorectal cancer via the miR-328-5p/E2F1 axis. Mol Ther. 2020;28:914-928. doi:10.1016/j.ymthe.2019.12.008
24. Shang A, Gu C, Wang W, et al. Exosomal circPACRGL promotes progression of colorectal cancer via the miR-142-3p/miR-506-3pTGF-beta1 axis. Mol Cancer. 2020;19:117. doi:10.1186/s12943020-01235-0

25. Dai J, Zhuang Y, Tang M, Qian Q, Chen JP. CircRNA UBAP2 facilitates the progression of colorectal cancer by regulating miR-199a/VEGFA pathway. Eur Rev Med Pharmacol Sci. 2020;24:7963-7971.

26. Verduci L, Strano S, Yarden Y, Blandino G. The circRNA-microRNA code: emerging implications for cancer diagnosis and treatment. Mol Oncol. 2019;13:669-680. doi:10.1002/1878-0261.12468

27. $\mathrm{Xu} \mathrm{T}$, Lei $\mathrm{T}$, Li SQ, Mai EH, Ding FH, Niu B. DNAH17-AS1 promotes pancreatic carcinoma by increasing PPME1 expression via inhibition of miR-432-5p. World J Gastroenterol. 2020;26: 1745-1757. doi:10.3748/wjg.v26.i15.1745

28. Cai Q, Zhao A, Ren LG, et al. MiR-425 involves in the development and progression of renal cell carcinoma by inhibiting E2F6. Eur Rev Med Pharmacol Sci. 2018;22:6300-6307.

29. Pei Y, Banerjee S, Sun Z, Jha HC, Saha A, Robertson ES. EBV nuclear antigen $3 \mathrm{C}$ mediates regulation of E2F6 to inhibit E2F1 transcription and promote cell proliferation. PLoS Pathog. 2016;12: e1005844. doi:10.1371/journal.ppat.1005844

30. Cheng FHC, Lin HY, Hwang TW, et al. E2F6 functions as a competing endogenous RNA, and transcriptional repressor, to promote ovarian cancer stemness. Cancer Sci. 2019;110:1085-1095. doi:10.1111/cas. 13920

\section{Publish your work in this journal}

Cancer Management and Research is an international, peer-reviewed open access journal focusing on cancer research and the optimal use of preventative and integrated treatment interventions to achieve improved outcomes, enhanced survival and quality of life for the cancer patient.
The manuscript management system is completely online and includes a very quick and fair peer-review system, which is all easy to use. Visit http://www.dovepress.com/testimonials.php to read real quotes from published authors. 